\title{
Ramsay Hunt Syndrome: Viral Infection Coexisting with COVID-19
}

\author{
Madhuri Mehta ${ }^{1}$ Akanksha Jain ${ }^{1} \quad$ Navroz Mehta ${ }^{2}$ \\ ${ }^{1}$ Department of Otorhinolaryngology, NC Jindal Institute of Medical \\ Sciences, Hisar, Haryana, India \\ 2Department of ENT, Guwahati Medical College, Guwahati, Assam, \\ India
}

\begin{abstract}
Address for correspondence Madhuri Mehta, MS, ENT, Department of Otorhinolaryngology, NC Jindal Institute of Medical Sciences, Hisar, Haryana 125005, India (e-mail: drmadhurimehta@gmail.com).
\end{abstract}

\begin{abstract}
Keywords

- COVID-19

- facial palsy

- sudden sensorineural hearing loss

Severe acute respiratory syndrome coronavirus 2 presents with a multitude of different symptoms and signs, most described is respiratory compromise. Many viral and bacterial infections coexist with coronavirus disease 2019 (COVID-19). Many cranial neuropathies have also been reported, including facial nerve palsy and sudden sensorineural hearing loss individually. Ramsay Hunt Syndrome, which is conventionally described as acute peripheral facial neuropathy, associated with erythematous vesicular rash of pinna or external auditory canal can at times present with cochlear or vestibular symptoms along with it. We report the case of a 32-year-old male who presented with Ramsay Hunt syndrome with cochlear manifestation during his COVID-19 illness. Evaluation of the patient and further management have been described.
\end{abstract}

\section{Introduction}

Severe acute respiratory syndrome coronavirus 2 (SARS CoV-2) is a newly discovered $\beta$ coronavirus that presents with a variety of different symptoms and signs. The most described presentation is respiratory compromise of varying degrees, others are diarrhea, anosmia, hypogeusia, etc. Nevertheless, the clinical picture of coronavirus disease 2019 (COVID-19) varies widely. Along with COVID-19, many other viral and bacterial infections have been found to coexist in a patient. Among many are also herpes and varicella-zoster virus (VZV). These viruses have been found to affect multiple sites with ear being one of them.

Various neuropathies both central and peripheral have been reported with SARS-CoV-2-related infection in recent studies. ${ }^{1}$ The mechanisms by which it causes cranial neuropathies have recently been hypothesized including neuroinvasion, neurovirulence, neurotropism, or autoimmune mechanisms. However, the exact pathogenesis is yet to be discovered. ${ }^{2}$

Published online

September 21, 2021
DOI https://doi.org/

$10.1055 / \mathrm{s}-0041-1735415$

ISSN 2581-9607
Isolated unilateral facial nervepalsyinSARS-CoV-2-positive patients has so far been described in many studies. ${ }^{1,3}$ Toscano et al described bilateral facial palsy in the context of GuillainBarre Syndrome with COVID-19. ${ }^{4}$

Adding to the plethora, many SARS-CoV-2 patients have been reported to present with sudden sensorineural hearing loss. ${ }^{5,6}$ Studies have believed that SARS-CoV-2 binds to the angiotensin-converting enzyme-2 receptor expressed in epithelial cells of the middle ear, stria vascularis, and spiral ganglion in mice. This causes an inflammatory response and increase in cytokines such as tumor necrosis factor- $\alpha$, interleukin-1, and interleukin-6. The mechanisms by which SARS-CoV-2 is believed to cause sensorineural hearing loss are attributed to both direct entry of virus into the cochlea and inflammation leading to cell stress. ${ }^{5}$ Mustafa compared the amplitude of transient-evoked otoacoustic emissions and latencies of vestibular evoked myogenic potential between asymptomatic COVID-19 real-time polymerase chain reaction (RT-PCR)-positive patients and normal subjects and
(C) 2021. Indian Society of Otology.

This is an open access article published by Thieme under the terms of the Creative Commons Attribution-NonDerivative-NonCommercial-License, permitting copying and reproduction so long as the original work is given appropriate credit. Contents may not be used for commercial purposes, or adapted, remixed, transformed or built upon. (https://creativecommons.org/licenses/by-nc-nd/4.0/).

Thieme Medical and Scientific Publishers Pvt. Ltd. A-12, 2nd Floor Sector 2, Noida-201301 UP, India 
found that COVID-19 infection have deleterious effects on cochlear hair cell functions. ${ }^{7}$

Till now, no publication in literature shows the existence of COVID-19 with both sensorineural hearing loss and facial palsy together. Here, we present a case of COVID-19 with Ramsay Hunt syndrome with sensorineural hearing loss and facial palsy.

\section{Case Report}

A 32-year-old male presented to ENT department of tertiary health-care center with left-sided facial weakness, left-sided hearing loss and tinnitus for the last 10 days, which were sudden in onset, progressive, and aggravated for the last 1 day to the extent that he was unable to communicate. He had a history of fever, sore throat, and vesicular eruptions over left pinna 12 days back for which he took treatment from other center. Two days later patient noticed facial weakness on left side as soon as he woke up. He noticed deviation of angle of mouth but was able to close his eyes with maximal effort. There were no complaints of reduced lacrimation or loss of taste sensation. He was brought to the emergency department and as part of institutional protocol underwent COVID-19 rapid antigen test. He tested positive for COVID-19 and opted for home isolation and was given medications accordingly. He was not referred to otorhinolaryngologist during this visit. He tested himself after 10 days for COVID-19 by RT-PCR method and was reported negative. During this time his facial weakness improved. On further history taking, patient revealed that he had decreased hearing from right side for more than 10 years and never consulted for it. He developed hard of hearing on left side for the last 10 days only and hence presented to our hospital. However, he had no complaints of ear discharge, vertigo, or history of any trauma.

He has no comorbidities at present and has a history of chickenpox infection in childhood. After the first episode of fever and vesicular eruptions, patient had taken tab acyclovir $800 \mathrm{mg}$ five times a day for 10 days.

On examination he was afebrile and vitals were stable. At rest, his face was symmetrical. He had loss of wrinkling of forehead on left side with complete eye closure with minimal effort. He was able to blow his cheek but asymmetry was noticed. A left-sided lower motor neuron grade III facial palsy (Houseman-Brackmann grading) was diagnosed (-Fig. 1). There was no other focal or systemic neurological deficit. He had lesions over left pinna, which were in healing phase and bilaterally intact tympanic membrane.

Patient was immediately investigated for pure tone audiometry (PTA), magnetic resonance imaging (MRI) brain and skull base, and high-resolution computed tomography (HRCT) temporal bone. The PTA revealed right-sided bone conduction hearing thresholds for $500,1,000 \mathrm{~Hz}$ to be $105 \mathrm{~dB}$ 2,000 and 4,000 $\mathrm{Hz}$ to be more than $120 \mathrm{~dB}$ and left-sided bone conduction hearing thresholds for $500,1,000,2,000$, and $4,000 \mathrm{~Hz}$ to be $80,80,80$, and $70 \mathrm{~dB}$, respectively, labeling it as right profound hearing loss with left severe sensorineural hearing loss ( - Fig. 2). MRI study showed no abnormality
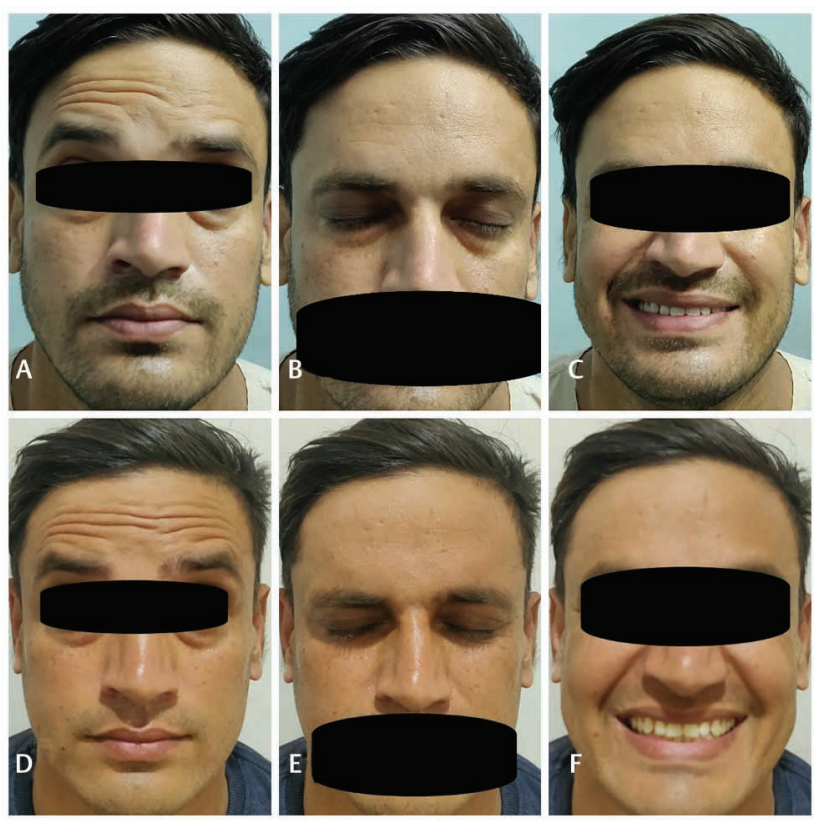

Fig. 1 Features of facial nerve functions on day 1 of presentation. (A) Loss of wrinkles over forehead on left side. (B) Complete eye closure with minimal effort. (C) Deviation of angle of mouth to right. Complete improvement in facial nerve function after 1 month. (D) Symmetrical wrinkling over forehead. (E) Complete eye closure with minimal effort. (F) symmetrical smile.

with bilateral normal internal auditory canal and VII to VIII nerve complexes. HRCT also revealed essentially normal study with facial canal dimensions within normal limits. His routine blood investigations were well within normal limits with marginally increased C-reactive protein levels. Immunoglobulin $\mathrm{M}$ and immunoglobulin $\mathrm{G}$ titers for herpes simplex virus were negative.

He was started on intravenous (IV) dexamethasone $8 \mathrm{mg}$ twice daily (BD), IV xanthinol nicotinate $1 \mathrm{~g}$ BD, and IV Neurobion Forte once daily with an antibiotic cover. He was also administered $0.5 \mathrm{~mL}$ intratympanic triamcinolone acetonide $40 \mathrm{mg} / \mathrm{mL}$ bilaterally on day 1, 3, and 5 under local anesthesia. However, patient showed only a $5 \mathrm{~dB}$ improvement in left hearing thresholds after first injection and no improvement after second and third injections. Speech discrimination score after all three intratympanic injections was $70 \%$ with the use of hearing aid bilaterally. His facial paresis improved completely on day 5 of admission with a slight improvement in tinnitus. He was discharged after 5 days on oral steroids and advised use of hearing aids. On subsequent examination after 7 days and 1 month, he had no improvement in unaided hearing.

\section{Discussion}

In 1907, James Ramsay Hunt postulated that the reactivation of latent VZV in the geniculate ganglion results in the combination of herpes zoster oticus and facial paralysis. In his original classification, he mentions that the facial nerve was always the first to be affected, in its sensory and/or motor portion, and later on auditory and/or vestibular symptoms 

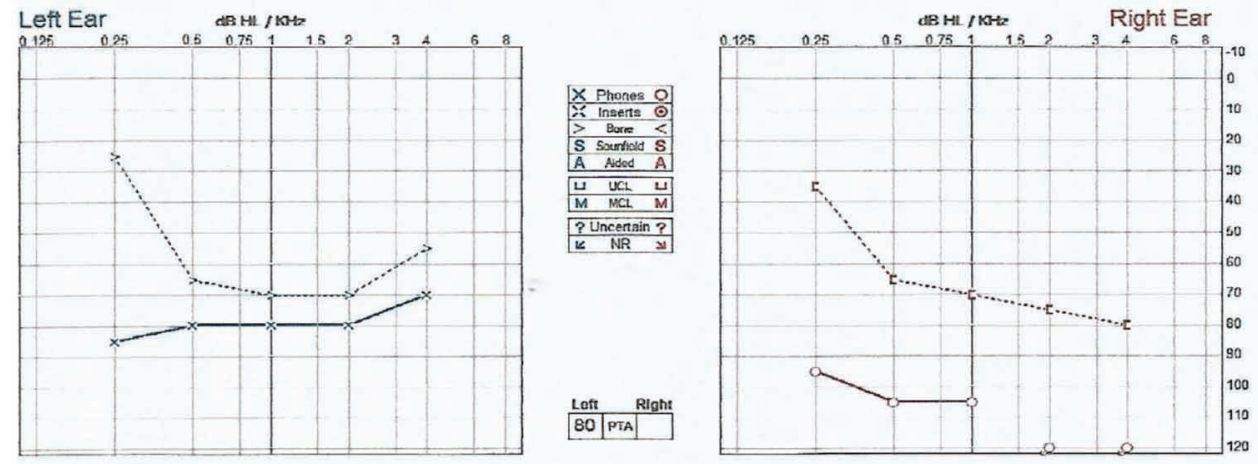

\section{DaY 1 PURE TONE AUDIOMETRY}
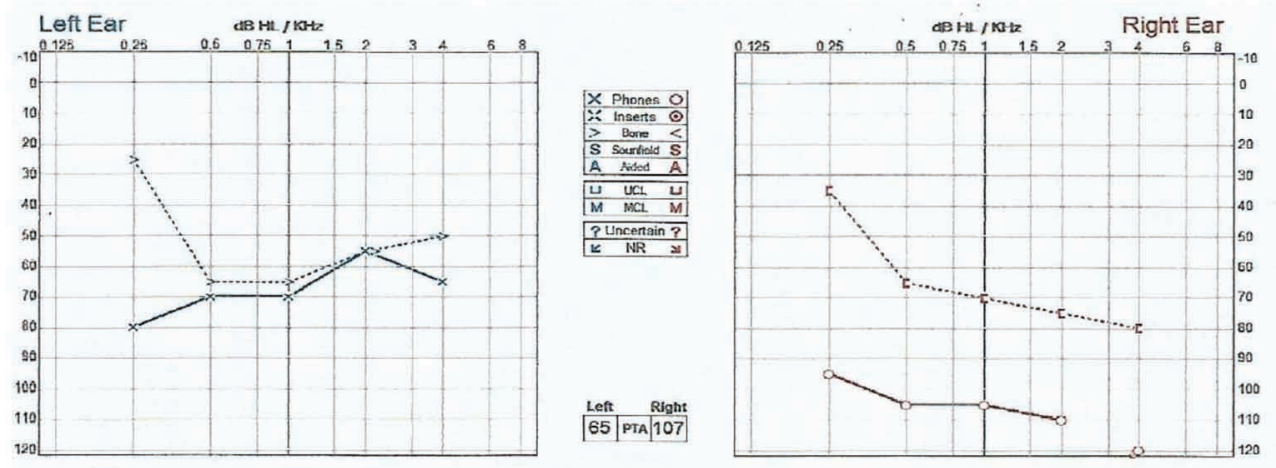

\section{MONTH PURE TONE AUDIOMETRY}

Fig. 2 Pure tone audiometry on day 1 of presentation and pure tone audiometry on 1 month follow-up.

could occur. Now, almost a century later, there is a lot of evidence in support of Hunt hypothesis. The presence of VZV genome has been demonstrated in the geniculate ganglion and also in the spiral and vestibular ganglion by using PCR. The presence of its genome indicates that VZV potentially infects the spiral and vestibular ganglia latently and that its reactivation may cause a cochleovestibular disorder. ${ }^{8}$

In the present case, infection with COVID-19 might have been the stress factor for reactivation of VZV.

López-Blanco et al reported a case with right facial paralysis, ipsilateral otalgia, and cutaneous eruption affecting the central area of the right ear and the right side of the soft palate and gait ataxia. ${ }^{9}$

Codeluppi et al compared the incidence and clinical features of individuals presenting in emergency rooms with facial palsy during the Italian COVID-19 outbreak and in the same period of 2019. They found that the incidence increased from 22 patients in 2019 to 38 in 2020 out of which only 2 of 2019 cohort and 8 of 2020 cohort tested positive for COVID-19. ${ }^{1}$

Lima et al presented a series of eight patients with isolated unilateral facial palsy with COVID-19. ${ }^{3}$ Similar cases have also been reported by Goh et al, ${ }^{10}$ Casas et al, ${ }^{11}$ Elkhouly et al. ${ }^{12}$ and in a pregnant female by Figueiredo et al. ${ }^{13}$ Other studies have demonstrated facial paralysis unilaterally or bilaterally with other neurologic diseases such as Guillain-Barre syndrome.

Isolated sudden sensorineural hearing loss has been reported with COVID-19 by Sriwijitalai and Wiwanitkit in April 2020, ${ }^{6}$ Degen et al, ${ }^{14}$ Abdel Rhman and Abdel Wahid et al, ${ }^{15}$ and Koumpa et al. ${ }^{5}$ All of them had improvements in hearing after proper treatment with intratympanic steroids.

Many cases of facial paralysis with COVID-19 and sudden sensorineural hearing loss with COVID-19 have been reported till now, but to the best of our knowledge this is the first case of Ramsay Hunt syndrome with cochlear and vestibular symptoms with COVID-19 infection.

\section{Conflict of Interest}

The authors declare that there is no conflict of interest.

\section{References}

1 Codeluppi L, Venturelli F, Rossi J, et al. Facial palsy during the COVID-19 pandemic. Brain Behav 2021;11(1):e01939. Doi: $10.1002 /$ brb3.1939

2 Costello F, Dalakas MC. Cranial neuropathies and COVID-19: neurotropism and autoimmunity. Neurology 2020;95(5): 195-196 
3 Lima MA, Silva MTT, Soares CN, et al. Peripheral facial nerve palsy associated with COVID-19. J Neurovirol 2020;26(6):941-944

4 Toscano G, Palmerini F, Ravaglia S, et al. Guillain-Barré syndrome associated with SARS-CoV-2. N Engl J Med 2020;382(26):2574-2576

5 Koumpa FS, Forde CT, Manjaly JG. Sudden irreversible hearing loss post COVID-19. BMJ Case Rep 2020;13(11):e23841910.1136/bcr-2020-

6 Sriwijitalai W, Wiwanitkit V. Hearing loss and COVID-19: a note. Am J Otolaryngol 2020;41(3):102473

7 Mustafa MW. Audiological profile of asymptomatic Covid-19 PCR-positive cases. Am J Otolaryngol 2020;41(3):102483

8 Kuhweide R, Van de Steene V, Vlaminck S, Casselman JW. Ramsay Hunt syndrome: pathophysiology of cochleovestibular symptoms. J Laryngol Otol 2002;116(10):844-848

9 López-Blanco R, Cazorla-Garcia R, Barbero-Bordallo N, Fernández-Ferro J. Neurological infections during the COVID19 pandemic. Neurologia 2020;35(4):273-274
10 Goh Y, Beh DLL, Makmur A, Somani J, Chan ACY. Pearls \& Oysters: facial nerve palsy in COVID-19 infection. Neurology 2020;95(8):364-367

11 Casas E, Barbosa A, Rubio-García E, et al. [Isolated peripheral facial paralysis in a patient with COVID-19]. Rev Neurol 2020;71(1):40-41

12 Elkhouly A, Kaplan AC. Retraction: Noteworthy Neurological Manifestations Associated With COVID-19 Infection. Cureus 2021;13(3):r26. Doi: 10.7759/cureus.r26.

13 Figueiredo R, Falcão V, Pinto MJ, Ramalho C. Peripheral facial paralysis as presenting symptom of COVID-19 in a pregnant woman. BMJ Case Rep 2020;13(8):e23714610.1136/ bcr-2020-237146

14 Degen C, Lenarz T, Willenborg K. Acute profound sensorineural hearing loss after COVID-19 pneumonia. Mayo Clin Proc 2020;95(8):1801-1803

15 Abdel Rhman S, Abdel Wahid A. COVID -19 and sudden sensorineural hearing loss, a case report. Otolaryngology Case Reports 2020;16:100198 